\title{
Mohamed Guenad, Sayyid Qutb. Itinéraire d'un théoricien de l'islamisme politique
}

Paris, L'Harmattan, coll. « Comprendre le Moyen-Orient », 2010, 135 p.

Brahim Labari

\section{OpenEdition}

\section{Journals}

Édition électronique

URL : http://journals.openedition.org/assr/25496

DOI : $10.4000 /$ assr.25496

ISSN : $1777-5825$

Éditeur

Éditions de l'EHESS

Édition imprimée

Date de publication : 30 décembre 2013

Pagination : 206

ISSN : 0335-5985

Référence électronique

Brahim Labari, « Mohamed Guenad, Sayyid Qutb. Itinéraire d'un théoricien de l'islamisme politique », Archives de sciences sociales des religions [En ligne], 164 | 2013, mis en ligne le 21 février 2014, consulté le 21 septembre 2020. URL : http://journals.openedition.org/assr/25496 ; DOI : https://doi.org/ 10.4000 /assr.25496

Ce document a été généré automatiquement le 21 septembre 2020.

(c) Archives de sciences sociales des religions 


\section{Mohamed Guenad, Sayyid Qutb. Itinéraire d'un théoricien de l'islamisme politique}

Paris, L'Harmattan, coll. « Comprendre le Moyen-Orient », 2010, 135 p.

Brahim Labari

\section{RÉFÉRENCE}

Mohamed Guenad, Sayyid Qutb. Itinéraire d'un théoricien de l'islamisme politique Paris, L'Harmattan, coll. « Comprendre le Moyen-Orient », 2010, 135 p. 
1 Mohammed Guenad, enseignantchercheur en sciences politiques, renoue dans cet ouvrage avec une démarche bien Mohammed GUENAD rodée, mais un tantinet marginale de la politologie, à savoir l'analyse d'un mouvement politico-religieux à l'aune de l'une de ses figures de proue.

2 Sayyid Qutb, né en 1906 et exécuté par pendaison en 1966, fait partie de ces penseurs au parcours dense qui a toutes les allures d'un touche-à-tout (il était tour à tour poète, éducateur, journaliste et critique littéraire) pour finir en dernière instance sur la voie d'un islam radical, étant enrôlé de son plein gré dans la confrérie des Frères musulmans fondée en 1926 par Hassan Al Bana. À cet égard, il est considéré par l'histoire des idées religieuses comme l'un des principaux théoriciens de l'islamisme politique en

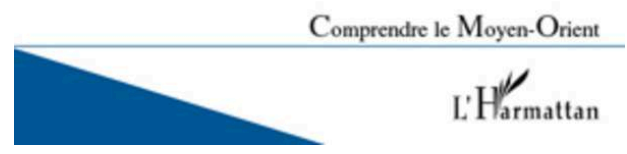
Égypte et dans le monde arabe.

\section{SAYYID QUTB}

\author{
Itinéraire d'un théoricien \\ de l'islamisme politique
}

'objectif proclamé de l'auteur est « d'entreprendre une étude d'ensemble sur la vie et les idées de Sayyid Qutb». M. Guenad s'assigne comme tâche d'expliquer et d'analyser les différentes étapes de la vie de Sayyid Qutb, de sa pensée et son évolution au cours du temps et des tensions qui ont pu accompagner ou orienter sa dynamique. Ainsi, il identifie différentes situations sociales et politiques des diverses époques dans le seul souci de clarifier une pensée, une prise de position ou la nature d'un engagement politique ou social.

Comment Sayyid Qutb s'est-il mué en idéologue islamiste dans les années 1950 ? Quels sont les facteurs l'ayant amené à changer de vision en maintes circonstances ?

Pour instruire ces interrogations de taille, l'auteur s'est employé à reconvoquer le contexte social et politique de l'Égypte d'avant la révolution de juillet 1952 au cours de laquelle les officiers libres mirent fin à la monarchie en Égypte. Et ce, pour décrypter cette mutation. Un tel contexte était tellement marqué par une ébullition socioculturelle que des penseurs, bons orateurs à la plume dénonciatrice, réussissent à s'engouffrer dans l'antisystème et à remplir cette fonction tribunitienne chère à Georges Lavau. En effet, le parcours de Qutb est emblématique d'une vie dédiée à la lutte dans la foulée des guerres d'indépendance dans les pays arabes et contre les régimes politiques considérés comme impies. La persistance dans cette fonction tribunitienne lui a valu une popularité chez ses coreligionnaires, voire de la vox populi et le coup de grâce, sa condamnation à mort dans l'Égypte nassérienne. Plus même, une certaine postérité voit en lui le digne théoricien des mouvements islamistes « modernes ». Que ce soit Khomeyni en Iran chiite, le Soudan actuel ou les mouvements islamistes transnationaux, les connexions avec quatre mille pages de production idéologique, que l'on peut résumer que tout doit être rapporté à la loi divine pour être validé, se font jour. 
6 L'auteur, ayant manifestement le goût de la synthèse, résume le parcours de Sayyid Qutb à l'aune des positions successives contenues dans ses écrits. En premier lieu, il s'est employé avec brio à faire une présentation du cadre sociohistorique qui a fait émerger la pensée islamiste radicale de Sayyid Qutb, en tant qu'idéologue du mouvement des Frères musulmans.En second lieu, par une analyse du discours de Qutb, l'auteur s'intéresse aux modalités de la radicalisation de la pensée du courant réformiste en Égypte et comment le contexte politique de l'époque participe de ce processus de radicalisation.

7 L'action de Sayyid Qutb a été décryptée en quatre moments spécifiques : 1 . sa vie et son engagement; 2. sa conception de l'Islam; 3 . les modalités et l'avenir du rapport à l'Occident; et enfin, 4 . son idée sur la société dite jâhilite (ère de l'ignorance) et sur la hâkimiyya (la gouvernance). Sa ruralité originelle, ses voyages, notamment en Amérique, qu'il exécrait comme étant un pays sans spiritualité - une sorte de nomadisme religieux - l'expérience carcérale, son combat contre les pouvoirs constitués ont forgé chez lui une personnalité charismatique et fort dogmatique.

8 Extrêmement documenté, car la bibliographie de Sayyid Qutb est des plus fournies ( «'Amérique que j'ai vue », « Repère sur le chemin », «Problèmes de civilisation »...), le livre est un condensé sur le parcours politicoreligieux de Sayyid Qutb, de son enfance paysanne à son initiation au savoir religieux, sans oublier son combat politique dénotant clairement, selon l'auteur, « la longue et laborieuse marche d'une société vers l'échec politique et culturel, notamment au plus fort de la transition de la société égyptienne vers le modernisme ».

9 Sayyid Qutb incarne parfaitement les aléas de cette période transitoire : entre 1920 et 1940, des forces contradictoires de la tradition et de la modernité dans la société égyptienne ont convergé à former sa personnalité et sa vision du monde. Nonobstant, force est de relever l'impact profond de la vie traditionnelle sur l'esprit et la vision du monde de Sayyid Qutb par la grande place qu'il consacre dans sa biographie aux pratiques religieuses populaires, à son éducation islamique et aux coutumes de son village. À un âge précoce, Qutb a acquis la maitrise de la culture arabo-musulmane traditionnelle, telles la mémorisation du Coran et l'excellente connaissance de la langue arabe, facteurs qui ont préparé le terrain pour être un membre très en vue dans le monde littéraire. Dans cette période transitoire, il faut relever également la rupture qui se développait dans le système éducatif égyptien entre ceux qui avaient reçu une éducation religieuse ( $k u t a ̂ b$ ) et ceux dont elle était résolument laïque (madrasa).

10 Dans les années 1920, après avoir quitté son village pour vivre chez son oncle, ses capacités à «imaginer " la nation égyptienne furent renforcées par ses expériences dans les institutions scolaires de la capitale qui lui fournirent l'occasion de forger des liens avec de nombreuses autres personnalités instruites et avisées en politique à l'instar de 'Aqqâd. À ce moment-là, Qutb a cultivé l'idée de la séparation entre la religion et la littérature qui s'exprimera dans ses écrits des années 1930 et des années 1940. Peut-être au prétexte que toute littérature dérive du péché...

11 Pendant le milieu des années 1930, il y avait une réaction répandue en Égypte contre l'occidentalisation effrénée et l'échec à réaliser l'indépendance de la nation. Cette réaction étendue de la prolifération des clubs et des associations musulmanes exprime des positions antioccidentales. Les écrits de Qutb ont bien restitué les incertitudes de cette période-là en dénigrant l'occidentalisation de la société égyptienne. Il a particulièrement usé de cet argument dans sa réfutation de Tâha Hussein, penseur à 
tendance modernisante, et de son ouvrage "L'avenir de la culture en l'Égypte » édité en 1938. En cette période, Qutb a commencé à s'intéresser sérieusement au Coran en tant que révélateur de l'harmonie entre le spirituel et le temporel tant il est vrai que l'islam " est un ordre intégré complet, dit-il, un axe fixe autour duquel tourne la vie dans un ordre précis [...] s'appliquant au mariage, à la nourriture, à l'habillement, aux contrats, à toute activité et travail, à toutes les relations sociales et commerciales, à tous les us et coutumes ".

Une raison presque certaine de la répugnance de Qutb pour la culture occidentale fut l'influence profonde qu'eurent sur lui la religion et les coutumes au cours de sa socialisation primaire. Les références dispersées dans les travaux qu'il a publiés suggèrent que, sous le vernis de la culture occidentale, les attaches et l'instruction modernes qu'il avait reçues, Qutb était un traditionaliste dont les sensibilités culturelles étaient ancrées dans l'univers de l'Égypte "villageoise » (aryâf). "Étant donné cette structure identitaire préexistante, il était peut-être naturel pour Qutb d'être hésitant face aux aspects culturels de la civilisation occidentale ", argue M. Guenad. Mais peut-être un autre facteur fut davantage décisif, lié aux crises politiques et économiques qu'affrontât l'Égypte dans les années 1930 et 1940.

Le résultat fut l'apparente incapacité des politiciens égyptiens à satisfaire les aspirations nationalistes qui se propageaient dans le peuple, au cours de la lutte contre les Anglais dans les années 1919-1922, aspirations qui restèrent profondément ancrées dans la conscience collective durant les années précédant la Seconde Guerre mondiale. Le contexte a été aussi reconnaissable à l'incapacité du régime en place à débarrasser l'Égypte de l'influence britannique et à entraver l'abîme entre les riches propriétaires qui dominaient au parlement et la masse des paysans et citadins pauvres dont la condition devenait de plus en plus criante.

Au début de 1948, Qutb commence à exprimer ses sentiments nationalistes en mettant fortement en avant le Coran, dont le contenu est remodelé en une discussion théologique autour du contexte égyptien contemporain de tensions politique et sociale, et spécifiquement la condition des inégalités sociales qui affectaient la société naissante. La solution, selon Qutb, est le retour à un ordre politique et légal inspiré du Coran, en réponse à la crise politique permanente en Égypte. Dans cette perspective, Qutb aurait participé à l'opposition dès la fin des années 1940, à la recherche d'une solution idéologique pour l'ordre économique et politique défaillant de l'Égypte. Qutb se rapproche du discours islamiste, déjà tenu vigoureusement par les Frères musulmans d'Égypte.

Il est clair que Sayyid Qutb adopta une position islamiste de façon progressive et tendit initialement à soutenir le concept laïc de la différence nationale (qui avait dominé ses écrits depuis les années 1930), plutôt que de le supplanter pour un ordre islamique pur et dur. Malgré son puissant appel à une autorité scripturaire et prophétique, l'intérêt premier de Qutb restait le même : améliorer l'identité d'une nation virtuelle contre les modèles différents et concurrents de l'Occident.

La poursuite de cet objectif apparaît dans la première partie du premier travail important de Qutb concernant l'Islam: "la Justice sociale en Islam» al-'Adâla alIjtimẩiyya fí al-Islâm, publié en 1949. Son intérêt pour le Coran n'était pas purement intellectuel, mais plutôt une nécessité "psychique et intellectuelle». Ses souffrances étaient beaucoup plus évidentes dans ses écrits journalistiques et sa poésie. La disparition de sa mère, sa relation amoureuse brisée et sa santé précaire, tous ces 
facteurs l'ont conduit à s'intéresser de plus en plus à la religion pour des raisons personnelles et afin de trouver des réponses aux problèmes dont souffrait sa société. Ce n'est qu'au milieu des années 1950, quand Qutb fut prisonnier politique, qu'il commença à faire siennes des idées rigoureusement théocentriques qui firent sa renommée en tant qu'idéologue islamiste. Tout se passe finalement comme si une véritable force créatrice émanait de la vulnérabilité de notre théoricien.

Sayyid Qutb. Itinéraire d'un théoricien de l'islamisme politique est assurément une lecture dans la vie et la pensée de Sayyid Qutb. Le moins que l'on puisse dire est que cette vie est plurielle et diffuse. L'ouvrage jette la lumière sur les facteurs de transformation intellectuelle de Qutb avant la révolution 1952 et son apparition comme l'un des principaux idéologues des Frères musulmans. Une vue d'ensemble de la vie de Qutb est présentée, ou plutôt son itinéraire. Ce qui a amené l'auteur à conclure que Qutb était un produit d'une société qui était passée par des dislocations politiques et culturelles à un moment où se produisait la transition de l'Égypte d'une société traditionnelle à une société moderne. À suivre M. Guenad, Sayyid Qutb était l'archétype du résistant au "désenchantement» du monde dans le contexte égyptien. Mais son radicalisme religieux l'a entraîné loin d'un débat contradictoire rationnel.

Deux formes d'objections peuvent être formulées à la lecture de ce livre. La première est que l'auteur aurait pu justifier plus avant son choix de Sayyid Qutb à un moment où l'Égypte des Frères musulmans comptait une pléiade de zaïms religieux. On appréciera que l'auteur, armé du bon sens et de la maîtrise de son sujet, retienne que tout parcours politicoreligieux est tributaire du contexte et en cela les hommes font l'Histoire sans avoir prise sur aucune de ses lois, car rien n'est écrit à l'avance. C'est justement ce point qui me semble d'un intérêt certain dans cet ouvrage. Tout se passe finalement comme si les pesanteurs de toutes sortes avaient eu raison de la liberté de l'acteur à façonner, y compris sa propre histoire. La seconde objection est que l'articulation entre l'histoire de vie de Sayyid Qutb avec le mouvement des Frères musulmans n'est pas suffisamment étayée : les différents écrits de Sayyid Qutb ne recoupent pas clairement les grands principes de la confrérie (lesquels par ailleurs ? Qui les a théorisés ? À quelles fins?...).

On doit savoir gré à $\mathrm{M}$. Guenad de livrer un travail dépassionné à l'heure même où un certain discours mène à des condamnations tous azimuts sans prendre le recul nécessaire et examiner avec rigueur les petites histoires qui font l'Histoire. 